\title{
Constitutive activation of ERK1/2 signaling protects against myocardial ischemia via inhibition of mitochondrial fragmentation in the aging heart
}

\author{
Qiang Zhao" ${ }^{1 \#}$, Fen Liu ${ }^{2 \#}$, Qian Zhao ${ }^{1}$, Jinyu Zhang ${ }^{1}$, Junyi Luo ${ }^{1}$, Xiaomei Li ${ }^{1}$, Yining Yang ${ }^{1,3}$ \\ ${ }^{1}$ Department of Cardiology, The First Affiliated Hospital of Xinjiang Medical University, Urumqi, China; ${ }^{2}$ Xinjiang Key Laboratory of \\ Cardiovascular Disease Research, Clinical Medicine Institute, The First Affiliated Hospital of Xinjiang Medical University, Urumqi, China; \\ ${ }^{3}$ Department of Cardiology, People's Hospital of Xinjiang Uygur Autonomous Region, Urumqi, China \\ Contributions: (I) Conception and design: Y Yang, Q Zhao, F Liu; (II) Administrative support: X Li; (III) Provision of study materials or patients: Q \\ Zhao, J Zhang, J Luo; (IV) Collection and assembly of data: Q Zhao, F Liu, Q Zhao, J Zhang; (V) Data analysis and interpretation: Q Zhao, F Liu, \\ Q Zhao; (VI) Manuscript writing: All authors; (VII) Final approval of manuscript: All authors. \\ \#These authors contributed equally to this work. \\ Correspondence to: Yining Yang. Department of Cardiology, The First Affiliated Hospital of Xinjiang Medical University, No. 137 Liyushan Road, \\ Urumqi 830054, China. Email: yangyn5126@163.com.
}

Background: Studies have shown that the ability of the myocardium to tolerate ischemia becomes significantly compromised with age. During ischemia, several endogenous protective signals are activated to protect the heart from injury, among which extracellular-signal regulated kinase (ERK) 1/2 signaling has been established as playing a pivotal role. However, in aging hearts, the activation of ERK1/2 is compromised. Mitogen-activated protein kinase/ERK kinase (MEK) is a major regulator of ERK1/2 signaling. In the present study, we investigated whether transduction of CaMEK, a constitutively activated MEK, using adeno-associated virus serotype 9 (AAV9) could protect the aging heart against ischemia.

Methods: Myocardial ischemia models were established in aging mice and senescent cardiomyocytes, and AAV9-mediated delivery of CaMEK was applied. Echocardiography, fluorescent staining, transmission electron microscopy, flow cytometry, and immunoblotting were used to explore the effects of CaMEK and their underlying mechanism.

Results: AAV9-CaMEK activated ERK1/2 signaling and exerted cardioprotective effects against ischemia in aging hearts. Specifically, CaMEK transduction decreased dynamin-related protein-1 (Drp1) expression and phosphorylation at serine 616, resulting in improved mitochondrial morphology and function in aging ischemic hearts. Furthermore, CaMEK transduction exerted similar protective effects in senescent cardiomyocytes under hypoxia. Meanwhile, with the inhibition of ERK1/2 signaling in senescent cardiomyocytes under hypoxia, the opposite effects were observed, including an increase in mitochondrial fragmentation and aggravation of mitochondrial dysfunction and cell apoptosis.

Conclusions: Our results suggested that AAV9-CaMEK alleviated ischemia-induced myocardium injury in the aging heart, at least in part, through inhibition of mitochondrial fragmentation. Therefore, AAV9CaMEK is a potential intervention for prevention of ischemia-induced injury of the aging myocardium.

Keywords: Aging; ERK1/2 signaling; myocardial ischemia; mitochondria; apoptosis

Submitted Jan 11, 2021. Accepted for publication Mar 24, 2021.

doi: $10.21037 / \mathrm{atm}-21-503$

View this article at: http://dx.doi.org/10.21037/atm-21-503 


\section{Introduction}

Ischemic heart disease ranks among the leading causes of global mortality, accounting for 9 million deaths each year (1). It can arise emergently as acute myocardial infarction (MI), in which coronary plaques rupture, causing an acute thrombotic occlusion of the coronary artery. As a consequence, the blood flow to the myocardium becomes restricted or blocked, thus depriving the myocardium of oxygen and nutrients, and resulting in the loss of cardiomyocytes. Aging is a major risk factor for ischemic heart disease (2). The prevalence of ischemic heart disease among individuals aged 75-79 years old is reported to be more than 30 times that in individuals aged 40-44 years old (3). The elevated risk of ischemic heart disease among the elderly population is associated with the fact that aging is accompanied by higher depression scores, a lower quality of life, less social support, less participation in formal cardiac rehabilitation, physical inactivity, and poorer health in general (4).

Studies on human and animal models of MI have demonstrated that the ability of the myocardium to tolerate ischemia becomes significantly compromised with aging (5-7). During ischemia, several endogenous protective signals are activated to protect the heart against injury, among which extracellular-signal regulated kinase (ERK) $1 / 2$ signaling and phosphatidylinositol-3-kinase signaling have been found to play pivotal roles (8-11). ERK1/2, which has vital involvement in the mitogen-activated protein kinase (MAPK) signaling pathway, generally promotes cell survival; however, under certain conditions, its activation can result in cell apoptosis (12). Studies have shown that ERK1/2 signaling activation is a potent strategy for protecting the heart against ischemic injury $(13,14)$. However, in aging mice, the activation of ERK1/2 signaling is impaired, which hinders their cardiac ability to tolerate ischemia (15). These observations suggest that activation of ERK1/2 signaling is a potential strategy for cardioprotection against ischemia in aging individuals.

MAPK/ERK kinase (MEK) is a major regulator of ERK1/2 signaling and can activate ERK1/2 transiently or persistently (16). Recently, constitutively activated MEK (CaMEK) was generated through removal of the coding sequence for amino acids $32-51$ and alteration of the nucleotides encoding the serine regulatory sites at 218 and 221 to glutamic and aspartic acid, respectively (17). CaMEK has been widely confirmed to specifically and effectively activate ERK1/2 signaling (18). However, whether CaMEK can protect the aging heart from ischemic injury has yet to be established.
Adenoviral gene therapy shows promise as a targeting treatment for cardiovascular disorders (19). Previously, it was found that adeno-associated virus serotype 9 (AAV9)cytomegalovirus (CMV) viral packaging was an efficient and safe tool for cardiac gene transfer (20). Accordingly, the current study was designed to investigate whether AAV9CMV-CaMEK could protect the myocardium of the aging heart against ischemia. We found that constitutive activation of ERK1/2 signaling using AAV9-CMV-CaMEK protected the aging heart against ischemia, suggesting the potential of AAV9-CMV-CaMEK as a strategy for cardioprotection in aging individuals. We present the following article in accordance with the ARRIVE reporting checklist (available at http://dx.doi.org/10.21037/atm-21-503).

\section{Methods}

\section{Ethics statement}

All animal experiments conformed to the Guide for the Care and Use of Laboratory Animals published by the National Institutes of Health. The experiments were approved by the Committee for the Care and Use of Laboratory Animals of the First Affiliated Hospital of Xinjiang Medical University, China (IACUC-20140214011).

\section{Reagents}

AAV9-CMV-CaMEK and its negative control (NC) vector were designed by our laboratory and produced by Virovek (Hayward, CA, USA). No-glucose Dulbecco's Modified Eagle Medium (DMEM), $0.25 \%$ trypsin solution, and fetal bovine serum (FBS) were obtained from Gibco Life Technologies (Grand Island, NY, USA). High-glucose DMEM, double resistance (penicillin and streptomycin), and phosphate-buffered saline (PBS) were purchased from HyClone (Logan, UT, USA). D-Galactose, PD98059, and dihydroethidium (DHE) were supplied by Sigma-Aldrich (St. Louis, MO, USA). JC-1 probe, Mito Tracker ${ }^{\mathrm{TM}}$ Green FM, MitoSOX ${ }^{\mathrm{TM}}$ Red, and a BCA-protein quantitation kit were purchased from Thermo Fisher Scientific (Waltham, MA, USA). RIPA lysis buffer, a cell counting kit-8 (CCK-8) analysis kit, and an adenosine triphosphate (ATP) testing kit, was obtained from Beyotime Institute of Biotechnology (Shanghai, China). Detection kits for lactate dehydrogenase $(\mathrm{LDH})$, creatine kinase (CK), and superoxide dismutase (SOD) were purchased from Nanjing Jiancheng Bioengineering Institute (Nanjing, Jiangsu, China). A senescence-associated $\beta$-galactosidase (SA- $\beta$-gal) detection 
kit, and antibodies against MEK, ERK1/2, phosphorylated ERK1/2 (p-ERK1/2), optic atrophy 1 (OPA1), dynaminrelated protein-1 (Drp1), and p-Drp1 Ser616 were obtained from Cell Signaling Technology (Beverly, MA, USA). Antibodies against p16, p21, mitofusin (Mfn) 1, Mfn2, p-Drp1 Ser637, mitochondrial fission protein 1 (Fis1), B-cell lymphoma-2 (Bcl-2), and BCL2 assoctiated X (Bax) were supplied by Abcam (Cambridge, MA, USA). The In Situ Cell Death Detection Fluorescein Kit was obtained from Roche Diagnostics Corp (Basel, Switzerland). The PE Annexin V Apoptosis Detection Kit I was obtained from BD Biosciences (San Jose, CA, USA). All other chemicals used were analytical reagents.

\section{Animals}

Young (12 to 16 weeks old) and aging (16 months old) male C57BL/6 mice supplied by Beijing Vital River Laboratory Animal Technology Co., Ltd. [License No. SCXK (Beijing) 2012-0001, Beijing, China] were used for the in vivo experiments. After an acclimation period of 1 week, mice were divided into an MI-CaMEK group and an MI-NC group and injected with AAV9-CMV-CaMEK and NC vector, respectively, at a multiplicity of infection (MOI) of $1 \times 10^{11} \mathrm{vg}$ per mouse via tail vein. Five weeks after the AAV9 injection, the young and aging mice in the MI groups were anesthetized using a mixture of ketamine $(100 \mathrm{mg} / \mathrm{kg})$, xylazine $(20 \mathrm{mg} / \mathrm{kg})$, and atropine $(1.2 \mathrm{mg} / \mathrm{kg})$, and thoracotomy and ligation of the left anterior descending coronary artery using a 7-0 nylon suture were performed as previously described $(21,22)$. Sham mice were subjected to all surgical procedures except the ligation. Animal model procedures were performed by a single experienced operator in a blinded manner. At 3 days after surgery, the cardiac function of the mice was tested, and the mice were sacrificed by intraperitoneal pentobarbital injection. Blood samples and cardiac tissues were collected for further experiments.

\section{Echocardiography}

The cardiac function of the mice was measured using echocardiography as described previously (23). Serial Doppler echocardiography was performed using the Vevo $^{\circledR} 3100$ ultrasound system with a $40-\mathrm{MHz}$ highfrequency ultrasonic probe (Fujifilm VisualSonics, Toronto, ON, Canada). Left ventricular end-diastolic diameter (LVEDD), left ventricular end-systolic diameter (LVESD), left ventricular end-diastolic volume (LVEDV), and left ventricular end-systolic volume (LVESV) were measured. Fractional shortening (FS) was calculated as (LVEDD LVESD)/LVEDD $\times 100 \%$, and left ventricular ejection fraction (LVEF) was calculated as (LVEDV - LVESV)/ LVEDV $\times 100 \%$.

\section{DHE staining}

Cardiac tissue from the mice was fixed in $4 \%$ paraformaldehyde solution for 4 hours, after which the tissue was transferred to a $20 \%$ sucrose solution for 12 hours. The tissue was then washed 3 times with PBS. Subsequently, the tissue was embedded in OCT embedding liquid and sectioned into 4- to 5- $\mu \mathrm{m}$-thick slices. The DHE staining solution $(10 \mu \mathrm{M})$ was added to the cold slices. After incubation for 30 minutes at $37^{\circ} \mathrm{C}$, the slices were washed 3 times with PBS. The fluorescence intensity was assessed using a fluorescence microscope (Carl Zeiss LSM800, Jena, Germany).

\section{Transmission electron microscopy}

Transmission electron microscopy was performed as described previously (24). Briefly, fresh cardiac tissue samples were fixed in $2.5 \%$ glutaraldehyde overnight at $4{ }^{\circ} \mathrm{C}$. After washing, the samples were postfixed in $1 \%$ osmium tetroxide for 2 hours before being dehydrated in a graded series of ethanol and acetone, and embedded in epoxy resin. The samples were then sliced, stained with uranyl acetate and lead citrate, and visualized with a transmission electron microscope (Hitachi H7500 TEM, Tokyo, Japan). Measurement of the mitochondrial area was performed using ImageJ software (National Institutes of Health, Bethesda, MA, USA).

\section{Terminal deoxynucleotidyl transferase dUTP nick end labeling staining}

Fresh-frozen heart sections of $5 \mu \mathrm{m}$ in thickness were stained using the In Situ Cell Death Detection Fluorescein Kit. Briefly, tissue sections were permeabilized with $0.1 \%$ Triton-100, washed 3 times with PBS, and incubated with the terminal deoxynucleotidyl transferase dUTP nick end labeling (TUNEL) reaction mixture for 1 hour at $37^{\circ} \mathrm{C}$ in the dark. Cell nuclei were stained with 4',6-diamidino-2phenylindoldihydrochloride (DAPI). Images of each slice were obtained using a laser scanning confocal microscope 
(Carl Zeiss LSM800, Jena, Germany). The apoptotic index was expressed as the ratio of the number of TUNELpositive nuclei to the total number of nuclei as previously described (21).

\section{Cell culture and treatments}

Primary cardiomyocytes were isolated from the hearts of neonatal Sprague Dawley rats (1-3 days old) as described previously (25-27). The primary cardiomyocytes were cultured in high-glucose DMEM with 10\% FBS and 1\% double resistance added at $37{ }^{\circ} \mathrm{C}$ in a fully humidified atmosphere containing $5 \% \mathrm{CO}_{2}$. Cardiomyocytes that grew steadily in 6-well plates were infected with AAV9-CMVCaMEK and NC vector at the MOI of $1 \times 10^{7} \mathrm{vg} /$ cell for 5 days. To induce senescence, cardiomyocytes were treated with $20 \mathrm{mg} / \mathrm{mL} \mathrm{D}$-galactose for 48 hours. Cardiomyocyte senescence was verified by SA- $\beta$-gal staining and the expression of $\mathrm{p} 16$ and $\mathrm{p} 21$ proteins.

To mimic ischemic injury, senescent cardiomyocytes were subjected to hypoxia. Briefly, cardiomyocytes were cultured in no-glucose DMEM and FBS in a BillupsRothenberg anoxic chamber (Del Mar, CA, USA). The anoxic chamber was filled with a gas mixture consisting of $5 \% \mathrm{CO}_{2}$ and $95 \% \mathrm{~N}_{2}$ at $37{ }^{\circ} \mathrm{C}$ for 24 hours (27). The senescent cardiomyocytes were assigned to 6 groups: (I) control group: senescent cardiomyocytes; (II) hypoxia blank group: senescent cardiomyocytes subjected to hypoxia; (III) hypoxia + NC group: senescent cardiomyocytes infected with $\mathrm{NC}$ vector and then subjected to hypoxia; (IV) hypoxia + CaMEK group: senescent cardiomyocytes infected with AAV9-CMV-CaMEK and then subjected to hypoxia; (V) hypoxia + dimethyl sulfoxide (DMSO) group: senescent cardiomyocytes treated with $0.1 \%$ DMSO and hypoxia; (VI) hypoxia + PD98059 group: senescent cardiomyocytes treated with $50 \mu \mathrm{M}$ PD98059, a specific inhibitor of ERK1/2 signaling, and hypoxia.

\section{Cell viability and apoptosis assay}

Cell viability was detected using a CCK-8 assay as instructed by the manufacturer. Cell apoptosis was detected using the PE Annexin V Apoptosis Detection Kit I. Treated cardiomyocytes were digested with $0.25 \%$ trypsin and collected in $1 \times$ binding buffer $\left(1 \times 10^{6}\right.$ cells $\left./ \mathrm{mL}\right)$. The cell suspension $(100 \mu \mathrm{L})$ was mixed with $5 \mu \mathrm{L} \mathrm{PE}$ Annexin V and $5 \mu \mathrm{L}$ 7-AAD. After being left in dark for 15 minutes, the supernatant was discarded, and the cells were washed with
$400 \mu \mathrm{L} 1 \times$ binding buffer. Cell apoptosis was measured using a flow cytometer (Beckman Coulter, Inc., Brea, CA, USA).

\section{Measurement of mitochondrial reactive oxygen species (ROS)}

Cardiomyocytes were cultured in a confocal petri dish. The cells were incubated with MitoSOX ${ }^{\mathrm{TM}} \mathrm{Red}$ dye solution $(10 \mu \mathrm{M})$ for 10 minutes at $37^{\circ} \mathrm{C}$ and then washed 3 times with PBS. Red fluorescence was measured with the laser scanning confocal microscope (excitation/emission: $488 \mathrm{~nm} />500 \mathrm{~nm})$.

\section{Assessment of mitochondrial morphology}

Mitochondrial morphology was assessed by performing MitoTracker staining as described previously (26). Cardiomyocytes cultured in a confocal petri dish were incubated with MitoTracker ${ }^{\mathrm{TM}}$ Green FM probe $(200 \mathrm{nM})$ in a $5 \% \mathrm{CO}_{2}$ incubator for 30 minutes at $37^{\circ} \mathrm{C}$. The cardiomyocytes were then washed 3 times with PBS and photographed with the laser scanning confocal microscope (excitation/emission: $488 \mathrm{~nm} />500 \mathrm{~nm}$ ). The number and individual area of mitochondria were measured using ImageJ software (National Institutes of Health, Bethesda, MA, USA).

\section{Measurement of mitochondrial membrane potential}

JC-1 fluorochrome was applied for measurement of the mitochondrial membrane potential of cells. JC-1 staining work solution $(5 \mu \mathrm{M})$ was added to a confocal petri dish containing treated cardiomyocytes. After incubation at $37^{\circ} \mathrm{C}$ for 20 minutes, the cells were washed 3 times with washing buffer. Then, red fluorescence and green fluorescence were assayed using the laser scanning confocal microscope (red fluorescence excitation/emission: $573 \mathrm{~nm} / 590 \mathrm{~nm}$; green fluorescence excitation/emission: $488 \mathrm{~nm} / 529 \mathrm{~nm}$ ). The mitochondrial membrane potential was presented as the ratio of red to green fluorescence intensity.

\section{Western blotting}

Mouse heart tissue and cardiomyocytes were lysed in RIPA lysis buffer, and the protein concentrations were determined with a BCA-protein quantitation kit. Proteins were separated on a sodium dodecyl sulfatepolyacrylamide gel electrophoresis (SDS-PAGE) system 
and then transferred onto polyvinylidene difluoride (PVDF) membranes (Millipore, Billerica, MA, USA). After the blocking of nonspecific binding sites with 5\% defatted milk in Tris-buffered saline for 2 hours at room temperature, the membrane was incubated with primary antibodies $(1: 1,000)$ overnight at $4{ }^{\circ} \mathrm{C}$. After incubation with secondary antibodies $(1: 5,000)$, the immunoblotting bands were visualized using an enhanced chemiluminescence kit (Millipore, Billerica, MA, USA) on the Omega Lum C Gel Imaging system (Aplegen, Pleasanton, CA, USA).

\section{Other biochemical analyses}

The levels of LDH, CK, and SOD activity, together with the ATP level, were analyzed using the corresponding commercial kits according to their operational protocols.

\section{Statistical analyses}

Data were expressed as mean \pm standard deviation (SD). Differences among groups were evaluated by one-way analysis of variance (ANOVA) followed by Tukey's post-hoc parametric test. Significant differences between 2 groups were assessed using unpaired $t$-tests. Statistical analyses were performed with SPSS version 19.0 software (SPSS, Inc., Chicago, IL, USA). $\mathrm{P}<0.05$ was considered to show a statistically significant difference.

\section{Results}

Constitutive activation of ERK1/2 signaling protected the aging heart against MI in vivo

ERK1/2 activation, as measured by phosphorylation, was increased in ischemic hearts of young mice (Figure 1A,B,C), demonstrating an endogenous protective mechanism of the heart in response to ischemia. However, in aging mice, this protective mechanism was impaired, as evidenced by a lack of significant difference in ERK1/2 activation between sham and ischemic hearts (Figure 1A,B,C). To test whether activation of ERK1/2 could protect the aging heart against ischemia, MEK was specifically overexpressed in the hearts of aging mice through the injection of AAV9-CMV-CaMEK (Figure 1D,E,F). Transduction of CaMEK was observed to activate ERK1/2 in aging mouse hearts (Figure 1D,E,F). However, echocardiography showed that CaMEK transduction had no significant effects on cardiac function in aging mice without MI (Figure 1G,H,I).
CaMEK transduction also activated ERK1/2 in aging hearts of mice with MI (Figure 17, K,L), which exerted a protective effect against ischemia by increasing LVEF and decreasing circulating concentrations of $\mathrm{LDH}$ and $\mathrm{CK}$ (Figure $1 M, N, O, P, Q)$. These results suggested that cardiac transduction of CaMEK in aging mice improves cardiac tolerance to ischemia.

\section{CaMEK transduction ameliorated cell apoptosis and improved mitochondrial morphology in aging hearts with MI}

As oxidative stress plays an important role in ischemiainduced cardiac dysfunction, the cardiac redox status was detected. Ischemia-induced oxidative stress, as evidenced by increased ROS and decreased SOD activity in aging hearts, was attenuated by CaMEK transduction (Figure $2 A, B, C$ ). Next, the mitochondrial function of the mice was evaluated. Ischemia decreased the cardiac ATP level, which was restored by CaMEK transduction (Figure 2D). Ischemia also induced cardiac mitochondrial fragmentation, and CaMEK transduction restored mitochondrial morphology in aging hearts, as revealed under an electron microscope (Figure 2E,F). Specifically, ischemia decreased the expression of $\mathrm{Mfn} 1$, and increased the content of Drp1, p-Drp1 at ser616, and Fis1 in aging hearts, while CaMEK transduction increased Mfn1 content and decreased the content of Drp1 and p-Drp1 at ser616 in aging hearts with MI (Figure $2 G, H, I, \mathcal{F}, K, L, M, N, O$ ). These observations suggested that CaMEK transduction improved mitochondrial morphology in aging hearts with MI, thus promoting myocardial survival. Ischemia decreased the Bcl2/Bax ratio in aging hearts, while CaMEK transduction increased the Bcl-2/Bax ratio in aging hearts with MI (Figure $2 P, Q)$. In addition, CaMEK transduction also decreased cell apoptosis in aging hearts with MI (Figure 2R,S). These results suggested that CaMEK inhibited cardiac mitochondrial fragmentation and apoptosis in aging mice.

\section{ERK1/2 activation protected senescent cardiomyocytes against bypoxia}

For further exploration of the molecular mechanism underlying the cardioprotective effect produced by CaMEK transduction, senescence of primary cardiomyocytes was induced through treatment with D-galactose. As shown in Figure $3 A, B, C, D, E$, D-galactose treatment increased the number of SA- $\beta$-gal-positive cells and increased the content of p16 and p21 in neonatal myocytes, showing a phenotype 


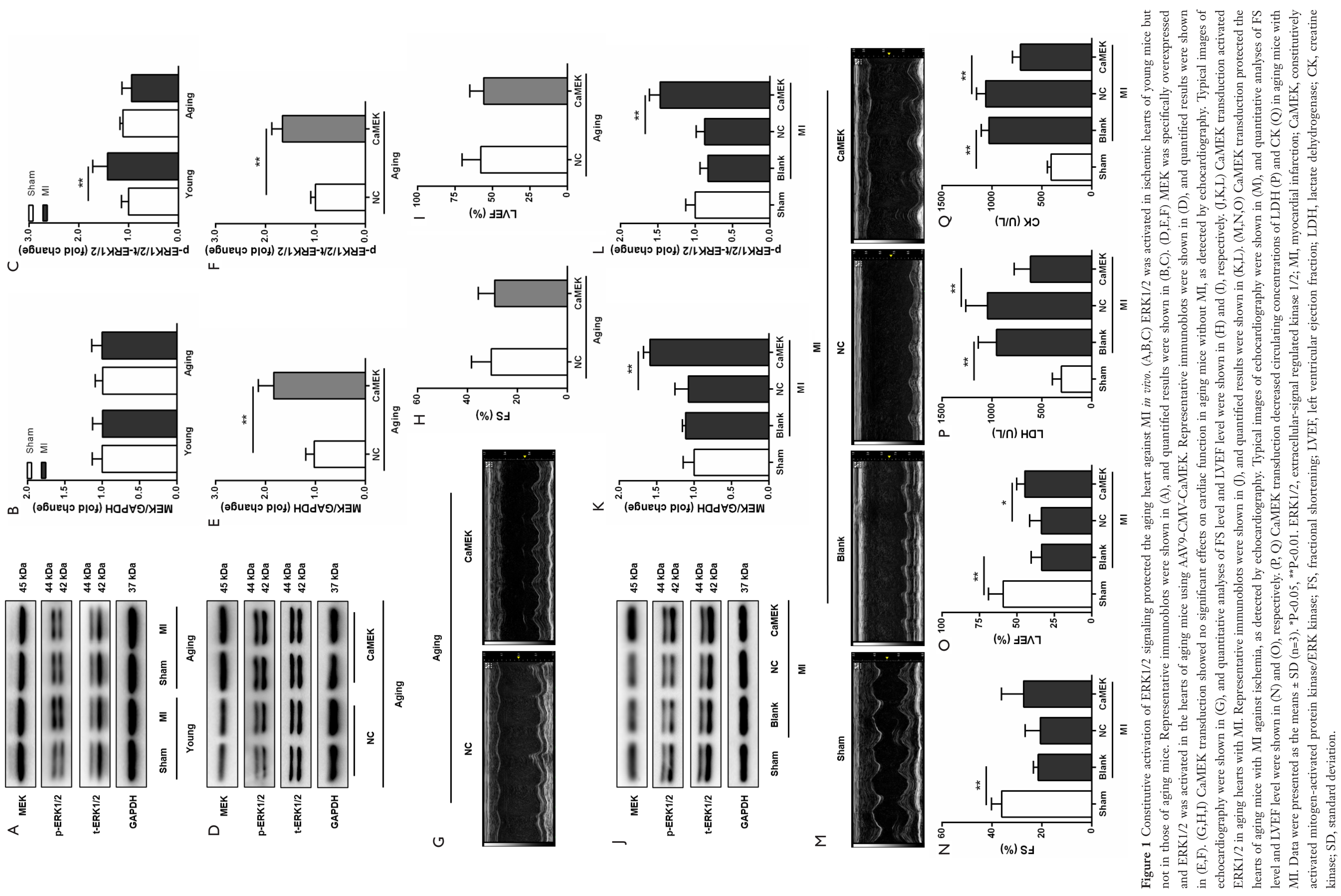



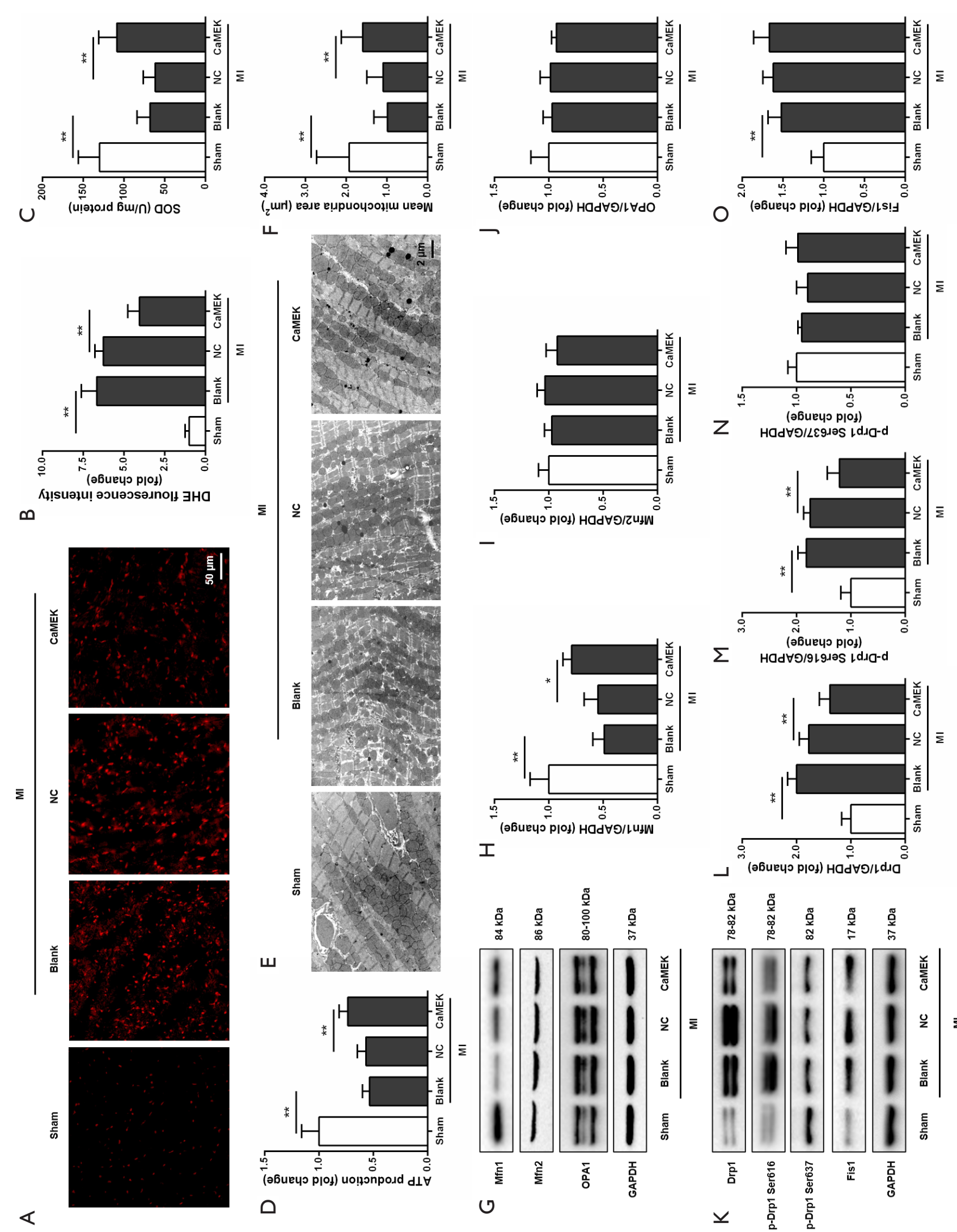
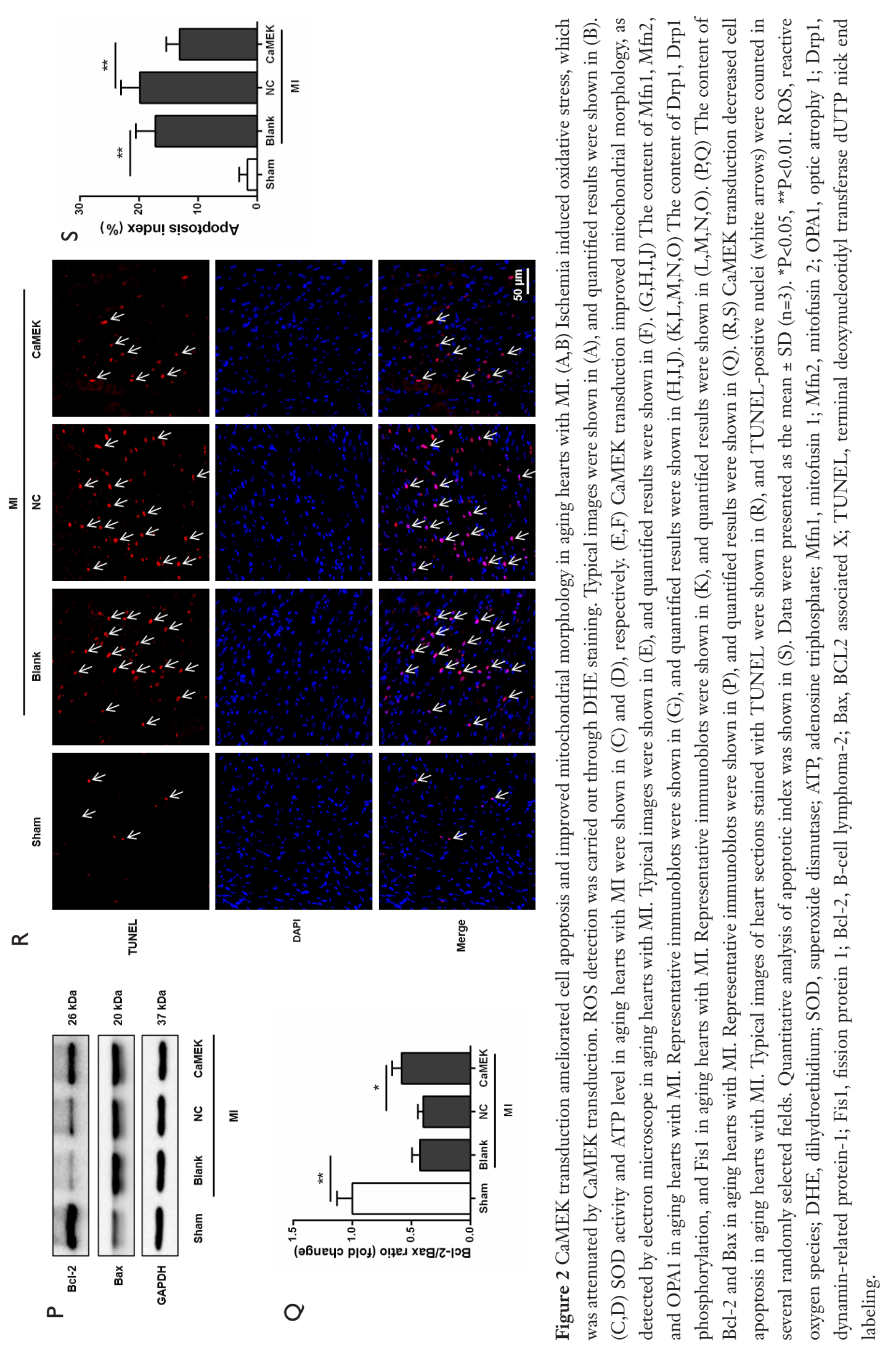

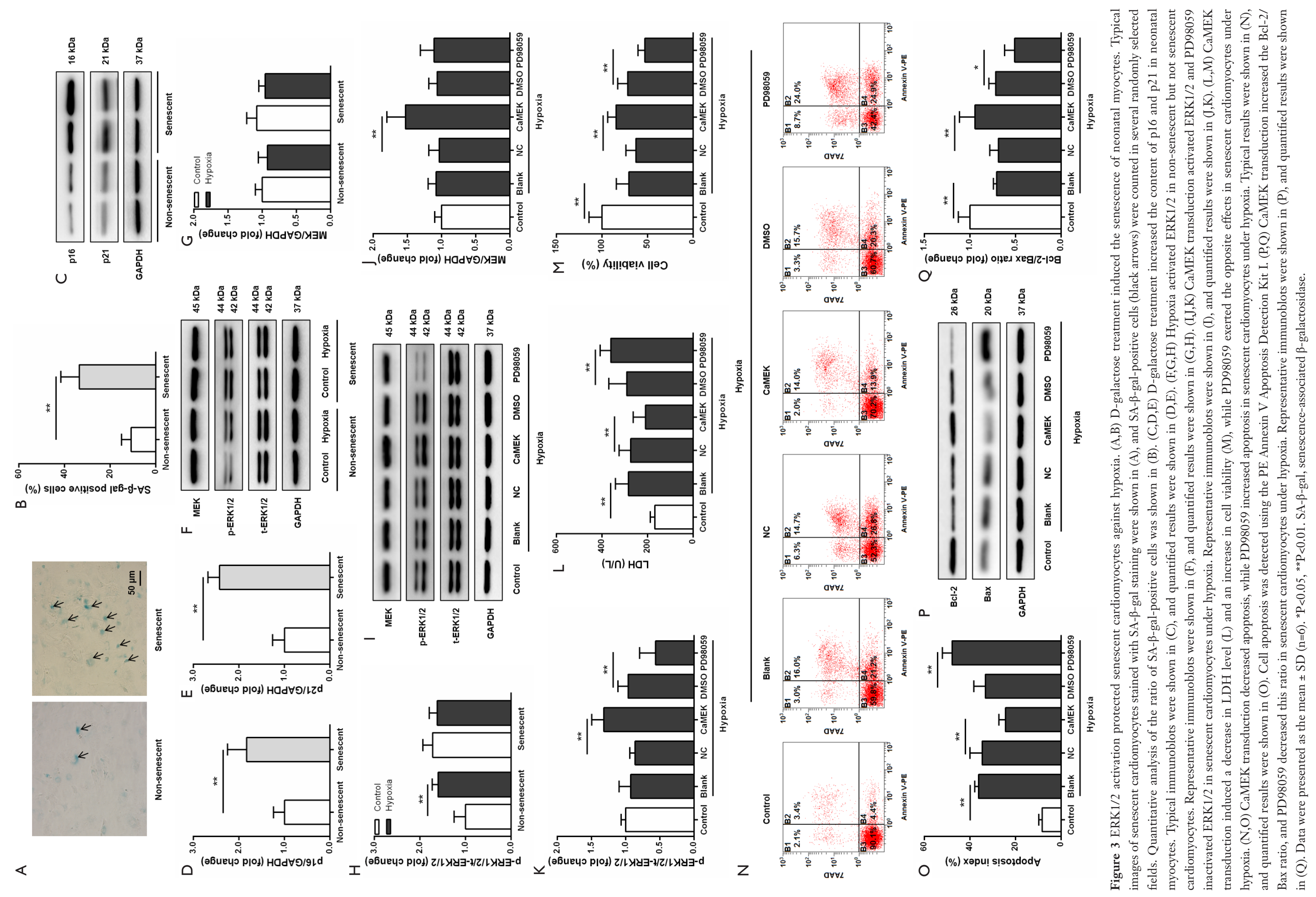
of senescent cardiomyocytes. Similar to the ischemic heart, hypoxia activated ERK1/2 in non-senescent but not senescent cardiomyocytes (Figure $3 F, G, H$ ). In senescent cardiomyocytes exposed to hypoxia, CaMEK transduction activated ERK1/2, whereas PD98059 inactivated ERK1/2 (Figure $3 I, \mathcal{F}, K)$. CaMEK transduction also induced a decrease in LDH levels and apoptosis but an increase in cell viability, while PD98059 exerted the opposite effects (Figure $3 L, M, N, O$ ). In addition, the $\mathrm{Bcl}-2 / \mathrm{Bax}$ ratio was increased by CaMEK transduction but decreased by PD98059 in senescent cardiomyocytes exposed to hypoxia (Figure 3P,Q). These results suggested that activation of ERK1/2 signaling protected senescent cardiomyocytes against hypoxia.

\section{ERK1/2 activation improved mitochondrial function in senescent cardiomyocytes under hypoxia}

Hypoxia increased the level of mitochondrial ROS, as detected by mitoSOX, and decreased SOD activity, the level of cellular ATP, and mitochondrial membrane potential in senescent cardiomyocytes (Figure 4A,B,C,D,E,F). In senescent cardiomyocytes under hypoxia, the activation of ERK1/2 induced by CaMEK transduction decreased the level of mitochondrial ROS, and increased SOD activity, the cellular ATP level, and mitochondrial membrane potential (Figure $4 A, B, C, D, E, F)$. Meanwhile, in senescent cardiomyocytes under hypoxia, inhibition of ERK1/2 by PD98059 increased the level of mitochondrial ROS, and decreased SOD activity, the cellular ATP level, and mitochondrial membrane potential (Figure $4 A, B, C, D, E, F$ ). These results suggested that ERK1/2 activation decreased the level of mitochondrial ROS and improved mitochondrial function in senescent cardiomyocytes under hypoxia.

To further evaluate mitochondrial function in senescent cardiomyocytes under hypoxia, the morphology of the mitochondria was observed. Hypoxia induced mitochondrial fragmentation, decreased the expression of $M \mathrm{fn} 1$, and increased the content of Drp1, p-Drp1 at ser616, and Fis1 in senescent cardiomyocytes (Figure $5 A, B, C, D, E, F, G, H, I, \mathcal{Z}, K, L$ ). Through its activation of ERK1/2, CaMEK transduction improved the mitochondrial morphology, increased the expression of Mfn1, and decreased the content of Drp1 and p-Drp1 at ser616 in senescent cardiomyocytes under hypoxia (Figure $5 A, B, C, D, E, F, G, H, I, \mathcal{F}, K, L)$. Meanwhile, ERK1/2 inhibition by PD98059 aggravated mitochondrial fragmentation, decreased Mfn1 expression, and increased the content of Drp1 and p-Drp1 at ser616 in senescent cardiomyocytes under hypoxia
(Figure $5 A, B, C, D, E, F, G, H, I, \mathcal{F}, K, L$ ). These findings reinforced the notion that the mitochondrial function of senescent cardiomyocytes under hypoxia was improved by ERK1/2 activation.

\section{Discussion}

Aging is a major risk factor for ischemic heart disease, which is associated with compromised myocardial tolerance to ischemia compounded by refractoriness to protective intervention (28). It is due to impaired activation of endogenous protective signals including ERK1/2, adenosine 5 '-monophosphate-activated protein kinase, and protein kinase C $(15,28,29)$. Because ERK1/2 has critical involvement in myocardial tolerance to ischemia and fails to be activated in response to ischemia in aging hearts, interventions which can activate ERK1/2 signaling are potential strategies for the treatment of ischemic heart disease. In the present study, we showed that AAV9-CMVCaMEK effectively activated ERK1/2 signaling, which protected aging hearts and senescent cardiomyocytes in mice against ischemia, at least in part, by improving mitochondrial morphology and function. These results suggest the potential of AAV9-CMV-CaMEK as a preventive tool for myocardial ischemic injury.

ERK1/2 signaling is involved in the regulation of cell proliferation, differentiation, survival, and apoptosis, and studies have previously described its role in ischemia and postconditioning $(30,31)$. ERK1/2 is involved in the reperfusion injury salvage kinase (RISK) pathway, which confers cardioprotection when activated $(32,33)$. The activation of ERK1/2 signaling protects the heart from ischemia reperfusion injury $(34,35)$. However, in aging hearts, the activation of ERK1/2 is compromised, which leads to the impairment of cardiac tolerance to ischemia. MEK, which is a major upstream activator of ERK1/2, phosphorylates the threonine-glutamic acid-tyrosine (TEY) motif within the activation loops of ERK1/2 (36). MEK has also been implicated in cardiac ischemic disease, in which it activates ERK1/2 signaling to protect the heart against ischemic injury (37). Our results showed that AAV9-CMV-CaMEK effectively upregulated MEK expression and activated ERK1/2 in aging hearts and senescent cardiomyocytes. Gene therapy has shown strong potential for the treatment of MI, peripheral arterial disease, restenosis after angioplasty, and rejection of heart transplantation (38). AAV is the most promising gene therapy vehicle due to its efficient infection of dividing and 
A

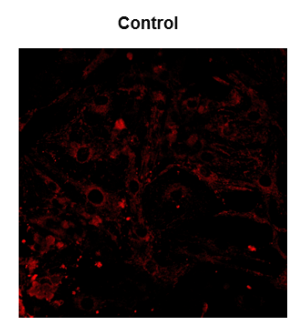

B

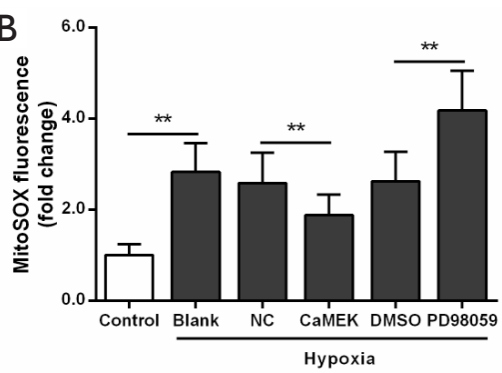

Hypoxia
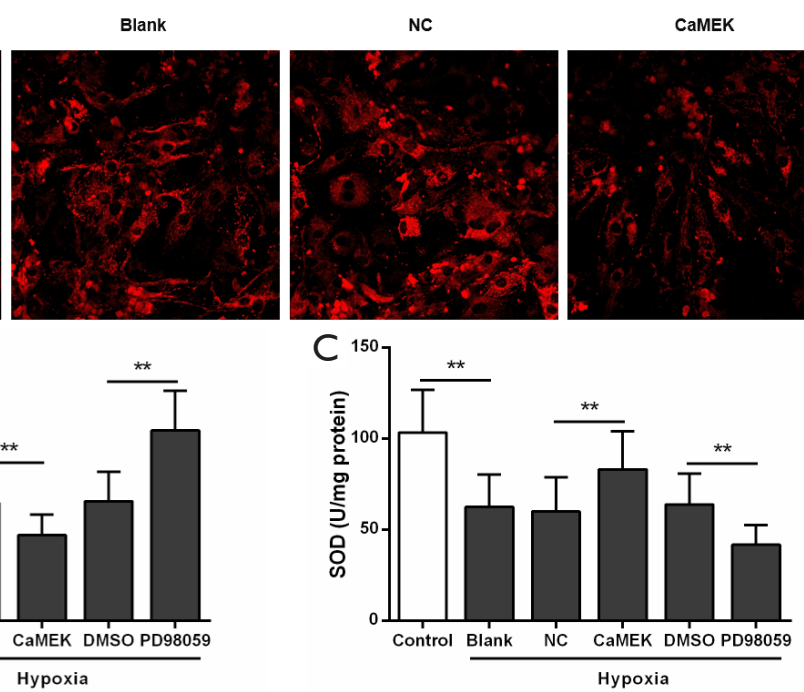

Hypoxia
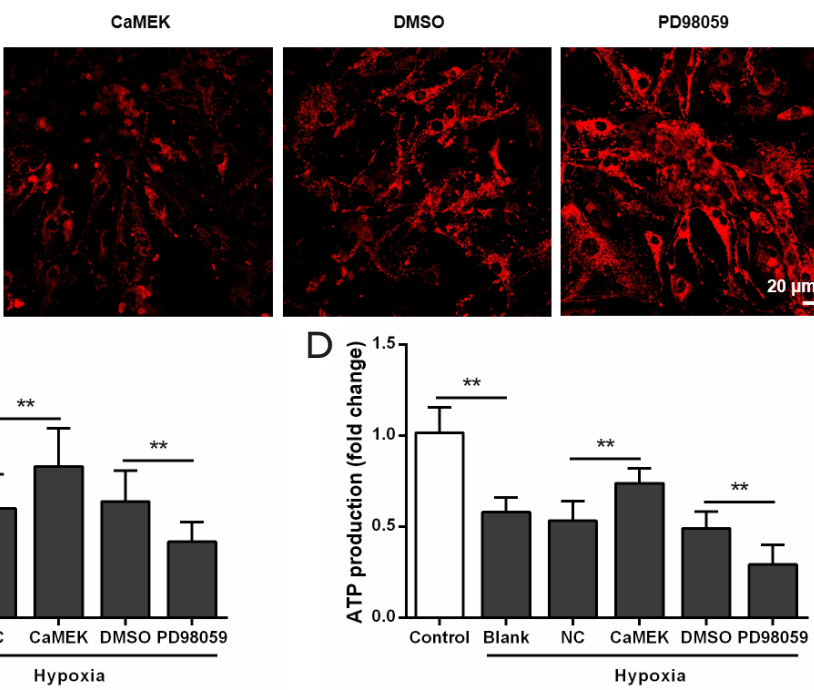

$E$

Control
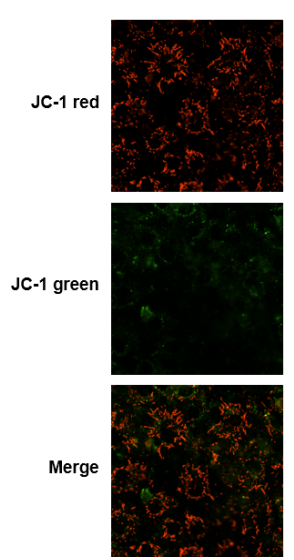
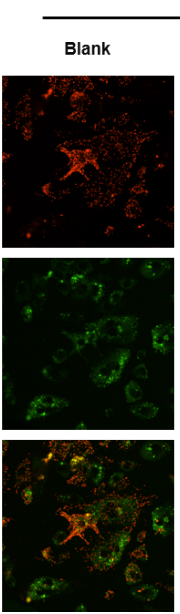

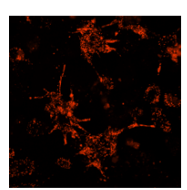

CaMEK
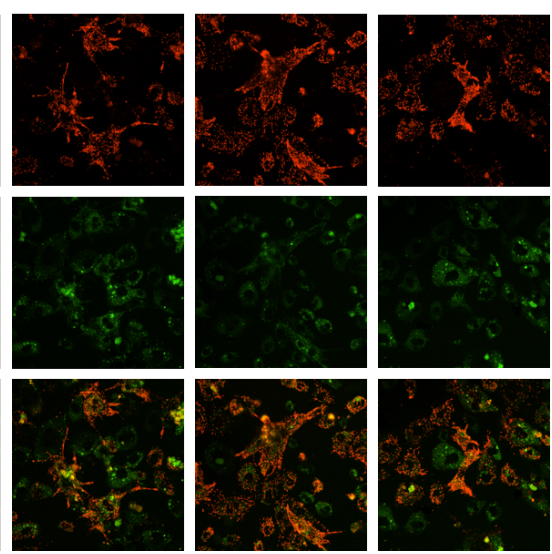

PD98059
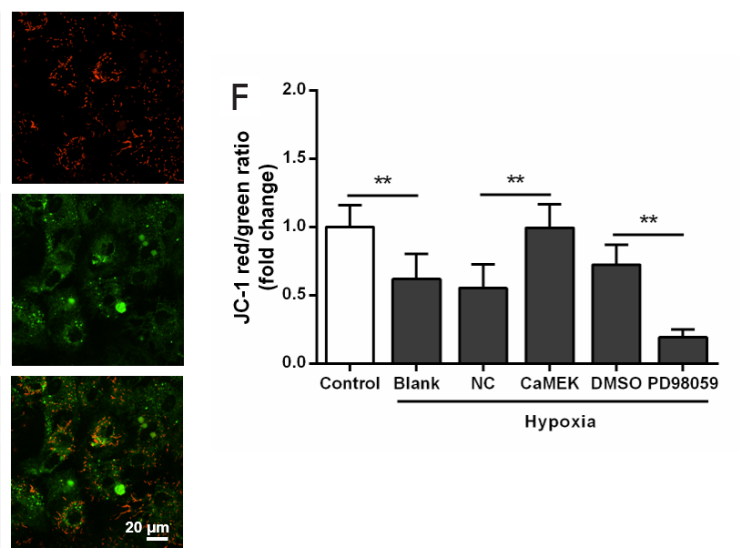

Figure 4 ERK1/2 activation improved mitochondrial function in senescent cardiomyocytes under hypoxia. (A,B) CaMEK transduction decreased mitochondrial ROS and PD98059 increased mitochondrial ROS in senescent cardiomyocytes under hypoxia, as detected by MitoSOX staining. Typical images of MitoSOX staining and quantified results were shown in shown in (A) and (B), respectively. (C,D) CaMEK transduction increased SOD activity (C) and ATP level (D), and PD98059 had the opposite effects in senescent cardiomyocytes under hypoxia. (E,F) CaMEK transduction increased mitochondrial membrane potential, while PD98059 decreased mitochondrial membrane potential in senescent cardiomyocytes under hypoxia. JC-1 staining was applied to detect mitochondrial membrane potential. Typical images were shown in (E), and quantified results were shown in $(\mathrm{F})$. Data were presented as the mean $\pm \mathrm{SD}(\mathrm{n}=6){ }^{*}{ }^{*} \mathrm{P}<0.01$.

non-dividing cells, with long-term gene expression and low toxicity (39). Our previous study found that AAV9-CMV was a more effective viral package than AAV9-CBA to direct cardiac gene transfer (20). This study reinforced the idea of $\mathrm{AAV}$-mediated gene therapy being a potent strategy to treat ischemic heart disease.

Mitochondria are essential organelles for integrating energy production, ROS generation, cellular signal transduction, and apoptosis (40). As the myocardium is a tissue with high energy demand, mitochondria play a pivotal role in maintaining optimal cardiac function. In contrast with other organelles, mitochondria are highly dynamic, constantly undergoing fusion and fission to maintain their function (41). Mitochondrial fusion and fission are regulated by several different guanosine triphosphatases. It is well established that mitochondrial outer and inner membrane 
A
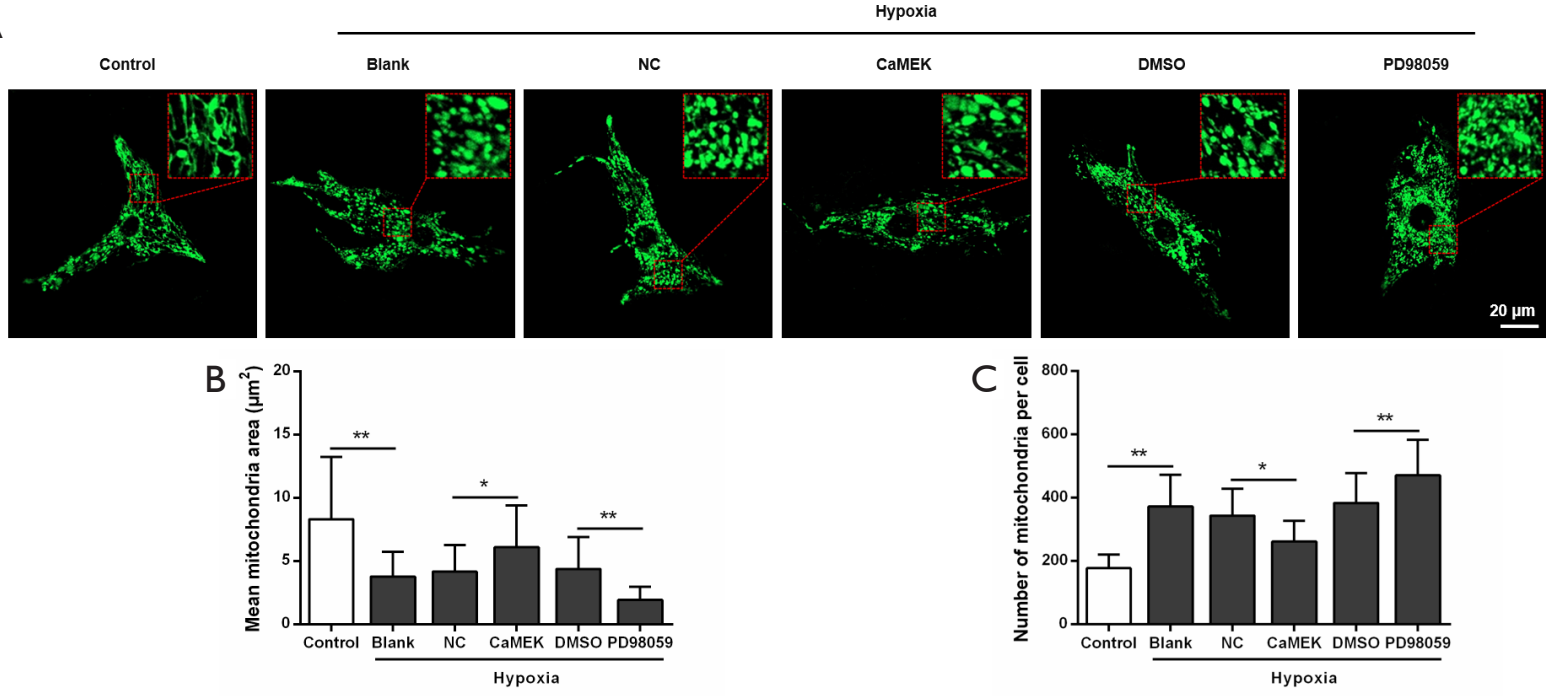

$C \overline{\bar{c}}$
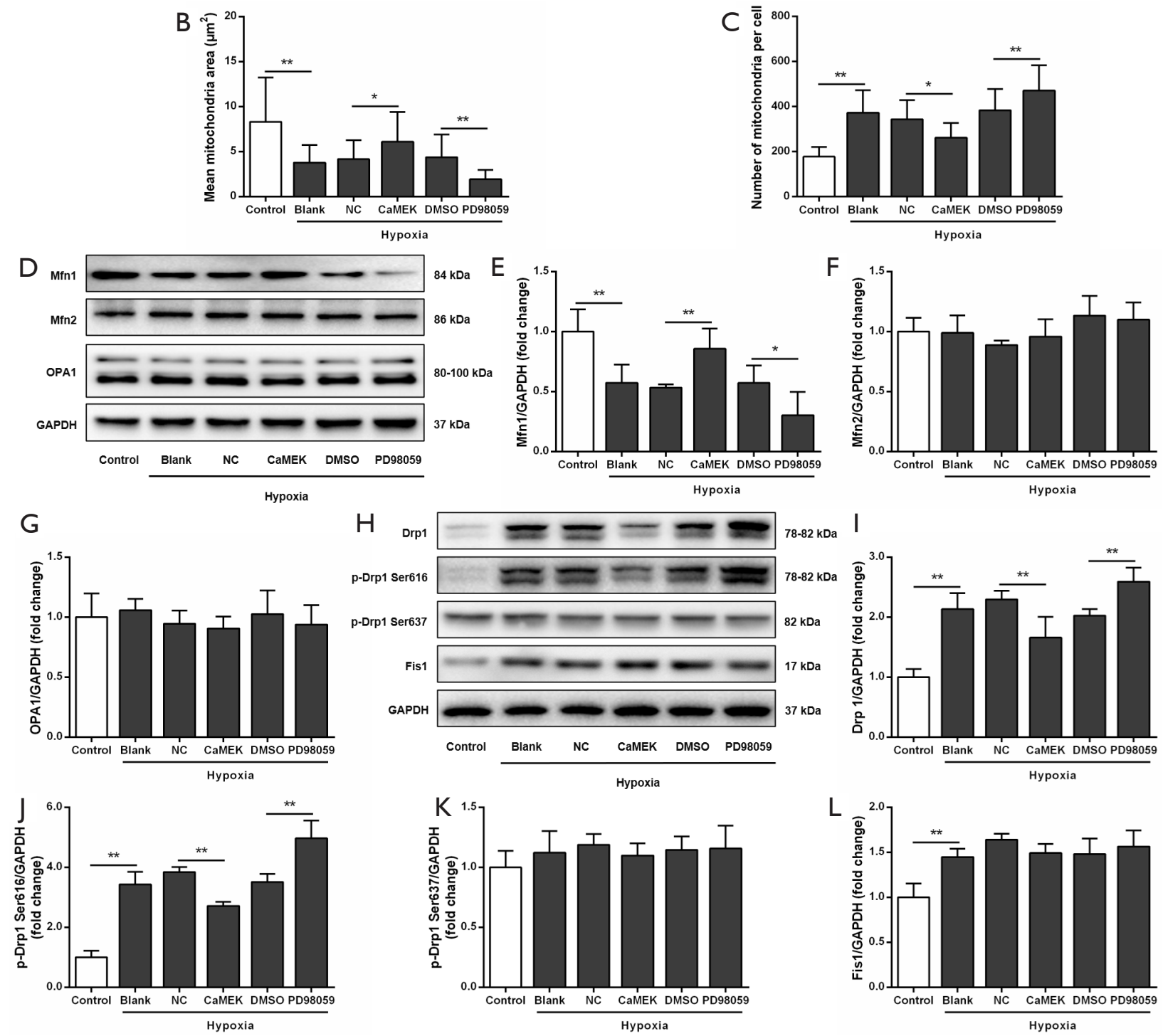

Figure 5 ERK1/2 activation improved mitochondrial morphology in senescent cardiomyocytes under hypoxia. (A,B,C) CaMEK transduction improved mitochondrial morphology and PD98059 aggravated mitochondrial fragmentation in senescent cardiomyocytes under hypoxia. Mitochondria were stained with MitoTracker probe. Typical images of mitochondrial morphology were shown in (A), and quantified results were shown in (B,C). (D,E,F,G) CaMEK transduction increased Mfn1 expression, and PD98059 decreased its expression in senescent cardiomyocytes under hypoxia. Representative immunoblots were shown in (D), and quantified results were shown in (E,F,G). (H,IJ,K,L) CaMEK transduction decreased Drp1 expression and phosphorylation at ser616, while PD98059 exerted the opposite effects on Drp1 level in senescent cardiomyocytes under hypoxia. Representative immunoblots were shown in $(\mathrm{H})$, and quantified results were shown in $(\mathrm{I}, \mathrm{J}, \mathrm{K}, \mathrm{L})$. Data were presented as the mean $\pm \mathrm{SD}(\mathrm{n}=6) .{ }^{*} \mathrm{P}<0.05,{ }^{* *} \mathrm{P}<0.01$. 
fusion is governed by mitofusins (Mfn1 and Mfn2) and OPA1, respectively (42). Mitochondrial fission is regulated by Drp1, which is recruited to the mitochondrial outer membrane through interaction with Fis1 and mitochondrial fission factor (43). Although Mfn1 and Mfn2 share approximately $77 \%$ sequence similarity, their functions in the promotion of mitochondrial fusion are distinct (44). Mfn1 is predominantly expressed over Mfn2 in the heart and testis (45). Phosphorylation of Drp1 at serine 616 activates mitochondrial fission, whereas phosphorylation of Drp1 at serine 637 inhibits mitochondrial fission $(46,47)$. Previous studies have demonstrated that Mfn1 and Drp1 are regulated by ERK1/2 signaling in the control of mitochondrial morphology and apoptosis $(48,49)$. In the present study, we found that in aging hearts with $\mathrm{MI}$ and in hypoxic senescent cardiomyocytes, AAV9-CMV-CaMEK increased the level of Mfn1 and decreased the content of Drp1 and p-Drp1 at Ser616 but did not significantly change the levels of Mfn2, OPA1, or Fis1, leading to improved mitochondrial morphology and function. An AAV9-CMVCaMEK-induced improvement in mitochondrial function was evidenced in aging hearts with MI by decreased mitochondrial ROS, and increased cellular ATP and mitochondrial membrane potential. These results indicated that activation of ERK1/2 signaling exerted beneficial effects on aging hearts and senescent cardiomyocytes by balancing mitochondrial fusion and fission in response to ischemia. Furthermore, mitochondria intimately communicate with the nucleus and endoplasmic reticulum, resulting in the regulation of calcium homeostasis and nuclear gene expression (50,51). Accumulating evidence has shown that increased nuclear and mitochondrial calcium accumulation occurred in the aging but not the mature heart, and these age-related differences were associated with increased DNA fragmentation and decreased cellular viability only in the aging heart (52). Recent data demonstrated that melatonin protected the heart from sepsis-induced myocardial injury by reducing oxidative damage, sustaining mitochondrial dynamics and calcium recycling as well as activations of cardioprotective signaling pathways including ERK1/2 signaling (53). Zhu et al. reported that daunomycin brought about apoptosis and DNA fragmentation accompanied by activation of ERK1/2 in cardiomyocytes, while cell apoptosis and DNA fragmentation became more prominent with pretreatment with PD98059 (54). Therefore, we supposed that apart from the regulation of mitochondrial fusion and fission, ERK1/2 signaling played crucial roles in developing a complex network between mitochondria, nucleus and other organelles in aging hearts under ischemia, and further studies are needed to investigate the various molecular mechanisms.

Taken together, the results of this study provided evidence that AAV9-CMV-CaMEK alleviated ischemiainduced myocardial injury in the aging heart. AAV9-CMVCaMEK activated MEK/ERK1/2 signaling, which inhibited mitochondrial fragmentation and decreased cell apoptosis in the hearts of aging mice with MI. These results suggest that maintaining a healthy mitochondrial function and the AAV9-CMV-CaMEK could be potential interventions for the prevention of ischemia-induced myocardium injury in aging individuals.

\section{Acknowledgments}

Funding: This study was supported by the National Natural Science Foundation of China (No. 81460069, 81700315, and 81770363).

\section{Footnote}

Reporting Checklist: The authors have completed the ARRIVE reporting checklist. Available at http://dx.doi. org/10.21037/atm-21-503

Data Sharing Statement: Available at http://dx.doi. org/10.21037/atm-21-503

Conflicts of Interest: All authors have completed the ICMJE uniform disclosure form (available at http://dx.doi. org/10.21037/atm-21-503). All authors report grants from National Natural Science Foundation of China, during the conduct of the study. The authors have no other conflicts of interest to declare.

Ethical Statement: The authors are accountable for all aspects of the work in ensuring that questions related to the accuracy or integrity of any part of the work are appropriately investigated and resolved. All animal experiments conformed to the Guide for the Care and Use of Laboratory Animals published by the National Institutes of Health. The experiments were approved by the Committee for the Care and Use of Laboratory Animals of the First Affiliated Hospital of Xinjiang Medical University, China (IACUC-20140214011).

Open Access Statement: This is an Open Access article 
distributed in accordance with the Creative Commons Attribution-NonCommercial-NoDerivs 4.0 International License (CC BY-NC-ND 4.0), which permits the noncommercial replication and distribution of the article with the strict proviso that no changes or edits are made and the original work is properly cited (including links to both the formal publication through the relevant DOI and the license). See: https://creativecommons.org/licenses/by-nc-nd/4.0/.

\section{References}

1. Ramachandra CJA, Hernandez-Resendiz S, Crespo-Avilan GE, et al. Mitochondria in acute myocardial infarction and cardioprotection. EBioMedicine 2020;57:102884.

2. Crimmins EM, Shim H, Zhang YS, et al. Differences between Men and Women in Mortality and the Health Dimensions of the Morbidity Process. Clin Chem 2019;65:135-45.

3. Roth GA, Johnson C, Abajobir A, et al. Global, Regional, and National Burden of Cardiovascular Diseases for 10 Causes, 1990 to 2015. J Am Coll Cardiol 2017;70:1-25.

4. Smilowitz NR, Mahajan AM, Roe MT, et al. Mortality of Myocardial Infarction by Sex, Age, and Obstructive Coronary Artery Disease Status in the ACTION RegistryGWTG (Acute Coronary Treatment and Intervention Outcomes Network Registry-Get With the Guidelines). Circ Cardiovasc Qual Outcomes 2017;10:e003443.

5. Mariani J, Ou R, Bailey M, et al. Tolerance to ischemia and hypoxia is reduced in aged human myocardium. J Thorac Cardiovasc Surg 2000;120:660-7.

6. Starnes JW, Bowles DK, Seiler KS. Myocardial injury after hypoxia in immature, adult and aged rats. Aging (Milano) 1997;9:268-76.

7. Lakatta EG, Sollott SJ. Perspectives on mammalian cardiovascular aging: humans to molecules. Comp Biochem Physiol A Mol Integr Physiol 2002;132:699-721.

8. Filippone SM, Samidurai A, Roh SK, et al. Reperfusion Therapy with Rapamycin Attenuates Myocardial Infarction through Activation of AKT and ERK. Oxid Med Cell Longev 2017;2017:4619720.

9. Liu Z, Chen JM, Huang H, et al. The protective effect of trimetazidine on myocardial ischemia/reperfusion injury through activating AMPK and ERK signaling pathway. Metabolism 2016;65:122-30.

10. Cheng CI, Lee YH, Chen PH, et al. Cobalt chloride induces RhoA/ROCK activation and remodeling effect in H9c2 cardiomyoblasts: Involvement of PI3K/Akt and MAPK pathways. Cell Signal 2017;36:25-33.
11. Lin RC, Weeks KL, Gao XM, et al. PI3K(p110 alpha) protects against myocardial infarction-induced heart failure: identification of PI3K-regulated miRNA and mRNA. Arterioscler Thromb Vasc Biol 2010;30:724-32.

12. Zhang DX, Ma DY, Yao ZQ, et al. ERK1/2/p53 and NF-kappaB dependent-PUMA activation involves in doxorubicin-induced cardiomyocyte apoptosis. Eur Rev Med Pharmacol Sci 2016;20:2435-42.

13. Patel VH, Karteris E, Chen J, et al. Functional cardiac orexin receptors: role of orexin-B/orexin 2 receptor in myocardial protection. Clin Sci (Lond) 2018;132:2547-64.

14. Chen Y, Ba L, Huang W, et al. Role of carvacrol in cardioprotection against myocardial ischemia/ reperfusion injury in rats through activation of MAPK/ ERK and Akt/eNOS signaling pathways. Eur J Pharmacol 2017;796:90-100.

15. Przyklenk K, Maynard M, Darling CE, et al. Aging mouse hearts are refractory to infarct size reduction with postconditioning. J Am Coll Cardiol 2008;51:1393-8.

16. Tanimura $S$, Takeda K. ERK signalling as a regulator of cell motility. J Biochem 2017;162:145-54.

17. Rubin J, Murphy TC, Zhu L, et al. Mechanical strain differentially regulates endothelial nitric-oxide synthase and receptor activator of nuclear kappa B ligand expression via ERK1/2 MAPK. J Biol Chem 2003;278:34018-25.

18. Gentile MT, Russo R, Pastorino O, et al. Ruta graveolens water extract inhibits cell-cell network formation in human umbilical endothelial cells via MEK-ERK1/2 pathway. Exp Cell Res 2018;364:50-8.

19. Schneider MD, French BA. The advent of adenovirus. Gene therapy for cardiovascular disease. Circulation 1993;88:1937-42.

20. Chen $\mathrm{BD}, \mathrm{He} \mathrm{CH}$, Chen $\mathrm{XC}$, et al. Targeting transgene to the heart and liver with AAV9 by different promoters. Clin Exp Pharmacol Physiol 2015;42:1108-17.

21. Ruze A, Chen BD, Liu F, et al. Macrophage migration inhibitory factor plays an essential role in ischemic preconditioning-mediated cardioprotection. Clin Sci (Lond) 2019;133:665-80.

22. Xiao C, Wang K, Xu Y, et al. Transplanted Mesenchymal Stem Cells Reduce Autophagic Flux in Infarcted Hearts via the Exosomal Transfer of miR-125b. Circ Res 2018;123:564-78.

23. Yang M, Song L, Wang L, et al. Deficiency of GATA3Positive Macrophages Improves Cardiac Function Following Myocardial Infarction or Pressure Overload Hypertrophy. J Am Coll Cardiol 2018;72:885-904.

24. Santulli G, Xie W, Reiken SR, et al. Mitochondrial calcium 
overload is a key determinant in heart failure. Proc Natl Acad Sci U S A 2015;112:11389-94.

25. Liu H, Shao Y, Qin W, et al. Myosin filament assembly onto myofibrils in live neonatal cardiomyocytes observed by TPEF-SHG microscopy. Cardiovasc Res 2013;97:262-70.

26. Zhao J, Gao JL, Zhu JX, et al. The different response of cardiomyocytes and cardiac fibroblasts to mitochondria inhibition and the underlying role of STAT3. Basic Res Cardiol 2019;114:12.

27. Han F, Chen Q, Su J, et al. MicroRNA-124 regulates cardiomyocyte apoptosis and myocardial infarction through targeting Dhcr24. J Mol Cell Cardiol 2019;132:178-88.

28. Peart JN, Pepe S, Reichelt ME, et al. Dysfunctional survival-signaling and stress-intolerance in aged murine and human myocardium. Exp Gerontol 2014;50:72-81.

29. Quan N, Wang L, Chen X, et al. Sestrin2 prevents age-related intolerance to post myocardial infarction via AMPK/PGC-1alpha pathway. J Mol Cell Cardiol 2018;115:170-8.

30. Inamura Y, Miyamae M, Sugioka S, et al. Sevoflurane postconditioning prevents activation of caspase 3 and 9 through antiapoptotic signaling after myocardial ischemiareperfusion. J Anesth 2010;24:215-24.

31. Chen HT, Yang CX, Li H, et al. Cardioprotection of sevoflurane postconditioning by activating extracellular signal-regulated kinase $1 / 2$ in isolated rat hearts. Acta Pharmacol Sin 2008;29:931-41.

32. Hernandez-Resendiz S, Roldan FJ, Correa F, et al. Postconditioning protects against reperfusion injury in hypertensive dilated cardiomyopathy by activating MEK/ ERK1/2 signaling. J Card Fail 2013;19:135-46.

33. Nduhirabandi F, Du Toit EF, Blackhurst D, et al. Chronic melatonin consumption prevents obesity-related metabolic abnormalities and protects the heart against myocardial ischemia and reperfusion injury in a prediabetic model of diet-induced obesity. J Pineal Res 2011;50:171-82.

34. Solan JL, Marquez-Rosado L, Lampe PD. Cx43 phosphorylation-mediated effects on ERK and Akt protect against ischemia reperfusion injury and alter the stability of the stress-inducible protein NDRG1. J Biol Chem 2019;294:11762-71.

35. Bei Y, Pan LL, Zhou Q, et al. Cathelicidin-related antimicrobial peptide protects against myocardial ischemia/reperfusion injury. BMC Med 2019;17:42.

36. Kehat I, Molkentin JD. Extracellular signal-regulated kinase 1/2 (ERK1/2) signaling in cardiac hypertrophy. Ann
N Y Acad Sci 2010;1188:96-102.

37. Chen Y, Liu F, Chen BD, et al. rAAV9-Mediated MEK1 Gene Expression Restores Post-conditioning Protection Against Ischemia Injury in Hypertrophic Myocardium. Cardiovasc Drugs Ther 2020;34:3-14.

38. Morishita R. Recent progress in gene therapy for cardiovascular disease. Circ J 2002;66:1077-86.

39. Skubis-Zegadlo J, Stachurska A, Malecki M. Vectrology of adeno-associated viruses (AAV). Med Wieku Rozwoj 2013;17:202-6.

40. Labbe K, Murley A, Nunnari J. Determinants and functions of mitochondrial behavior. Annu Rev Cell Dev Biol 2014;30:357-91.

41. Youle RJ, van der Bliek AM. Mitochondrial fission, fusion, and stress. Science 2012;337:1062-5.

42. Wu S, Lu Q, Wang Q, et al. Binding of FUN14 Domain Containing 1 With Inositol 1,4,5-Trisphosphate Receptor in Mitochondria-Associated Endoplasmic Reticulum Membranes Maintains Mitochondrial Dynamics and Function in Hearts in Vivo. Circulation 2017;136:2248-66.

43. Jones E, Gaytan N, Garcia I, et al. A threshold of transmembrane potential is required for mitochondrial dynamic balance mediated by DRP1 and OMA1. Cell Mol Life Sci 2017;74:1347-63.

44. Ikeda Y, Shirakabe A, Brady C, et al. Molecular mechanisms mediating mitochondrial dynamics and mitophagy and their functional roles in the cardiovascular system. J Mol Cell Cardiol 2015;78:116-22.

45. Spence SJ, Sharifi P, Wiznitzer M. Autism spectrum disorder: screening, diagnosis, and medical evaluation. Semin Pediatr Neurol 2004;11:186-95.

46. Li F, Fan X, Zhang Y, et al. Inhibition of myosin IIAactin interaction prevents ischemia/reperfusion induced cardiomyocytes apoptosis through modulating PINK1/ Parkin pathway and mitochondrial fission. Int J Cardiol 2018;271:211-8.

47. Din S, Mason M, Volkers M, et al. Pim-1 preserves mitochondrial morphology by inhibiting dynaminrelated protein 1 translocation. Proc Natl Acad Sci U S A 2013;110:5969-74.

48. Huang CY, Lai CH, Kuo CH, et al. Inhibition of ERKDrp1 signaling and mitochondria fragmentation alleviates IGF-IIR-induced mitochondria dysfunction during heart failure. J Mol Cell Cardiol 2018;122:58-68.

49. Pyakurel A, Savoia C, Hess D, et al. Extracellular regulated kinase phosphorylates mitofusin 1 to control mitochondrial morphology and apoptosis. Mol Cell 2015;58:244-54.

50. Kim KH, Son JM, Benayoun BA, et al. The Mitochondrial- 
Encoded Peptide MOTS-c Translocates to the Nucleus to Regulate Nuclear Gene Expression in Response to Metabolic Stress. Cell Metab 2018;28:516-24.e7.

51. Soledad RB, Charles S, Samarjit D. The secret messages between mitochondria and nucleus in muscle cell biology. Arch Biochem Biophys 2019;666:52-62.

52. Faulk EA, McCully JD, Tsukube T, et al. Myocardial mitochondrial calcium accumulation modulates nuclear calcium accumulation and DNA fragmentation. Ann

Cite this article as: Zhao Q, Liu F, Zhao Q, Zhang J, Luo J, Li X, Yang Y. Constitutive activation of ERK1/2 signaling protects against myocardial ischemia via inhibition of mitochondrial fragmentation in the aging heart. Ann Transl Med 2021;9(6):479. doi: 10.21037/atm-21-503
Thorac Surg 1995;60:338-44.

53. Zhong J, Tan Y, Lu J, et al. Therapeutic contribution of melatonin to the treatment of septic cardiomyopathy: A novel mechanism linking Ripk3-modified mitochondrial performance and endoplasmic reticulum function. Redox Biol 2019;26:101287.

54. Zhu W, Zou Y, Aikawa R, et al. MAPK superfamily plays an important role in daunomycin-induced apoptosis of cardiac myocytes. Circulation 1999;100:2100-7. 